\title{
The Management of Pediatric Heart Failure: Current State and Future Managements
}

\author{
Erhan Hafiz ${ }^{1}$, Dogan Kahraman ${ }^{1}$, Emjed Khali2 and Omer Faruk DOGAN ${ }^{3 *}$ \\ ${ }^{1}$ Department of Cardiovascular Surgery, Gaziantep University School of Medicine, Gaziantep, Turkey \\ ${ }^{2}$ Dr. Ersin Arslan Resesrch and Training Hospital, Dept. Of Cardiovascular Surgery, Gaziantep, Turkey \\ ${ }^{3}$ Department of Cardiovascular Surgery, Adana Numune Education and Training Center, Adana, Turkey
}

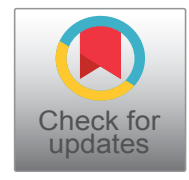

*Corresponding author: Omer Faruk DOGAN, Department of Cardiovascular Surgery, Adana Numune Education and Training Center, Adana, Turkey, E-mail: ofdogan@hacettepe.edu.tr

\section{Introduction}

As we know that heart failure is a complex pathology that may be seen in children with congenital heart disease include development of cardiomyopathies due to myocarditis. In general, this severe clinical condition is associated with a high rate of morbidity and mortality and places a significant burden on families. Current medical and/or surgical treatment modalities are taken largely from the management of heart failure in adults, though clear survival benefit of these medications are lacking. There is no adequate published data on the overall prevalence or incidence of heart failure in children. However, the success of mechanical circulatory support in management of heart failure has raised the prospect of a previously unavailable treatment modality. Heart transplant remains the gold standard treatment, but the number of patients requiring this treatment far outweighs the donor availability. It is therefore not surprising to see the popularity of various circulatory support modalities, with devices ranging from veno-arterial extracorporeal membrane oxygenation (ECMO) to ventricular assist devices which have different properties such as pulsatile or continuous flow management. Indication, timing and the choice of the type of mechanical support are critical in order to avoid lethal complications. In many patients we can see that hemorrhage due to continous anticoagulation requrement, thromboembolism and infections. In pediatric patients with heart failure, mechanical supporting system is used rarely for bridge to transplantation in many of centers. Active research is currently underway to develop newer and more durable devices that will assist the pediatric population across all age groups. The combined experience developed through the usage of different devices in pediatric and adult populations has led to the application of mechanical circulatory support in some subgroups of grown-up congenital heart diseases patients, particularly those with systemic right ventricular failure. Left and/ or right ventricular assist programs and/or the use of paracorporeal supporting devices combined with inodilators and VADs have taken an increasingly main role in the management of advanced heart failure in children. The predominant devices has been used for bridge to heart transplantation. Excellent results are currently achieved for most children who requiring surgery. There is an ongoing investigation to improve outcomes in highrisk populations, such as small infants and those with complex congenital heart disease. Additionally, there is an active investigation and interest in expansion of VADs beyond the predominant utilization as a bridge to a heart transplant into ventricular recovery, device explant without a heart transplantation (bridge to recovery), and placement of devices without the expectation of destination therapy. Survival four years after hospital admission has been reported as only $70 \%$. In the United States, it is currently estimated that greater than five million adults have heart failure with projections reaching greater than eight million by 2030 . The mortality at five-years after the diagnosis of heart failure remains at approximately $50 \%$. The costs associated with disease is staggering, with estimates that the total annual cost

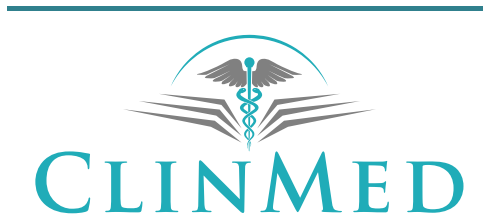

Citation: Hafiz E, Kahraman D, Khalil E, Omer FD (2018) The Management of Pediatric Heart Failure: Current State and Future Managements. Int J Surg Res Pract 5:074. doi.org/10.23937/2378-3397/1410074 Accepted: May 26, 2018; Published: May 28, 2018

Copyright: (C) 2018 Hafiz E, et al. This is an open-access article distributed under the terms of the Creative Commons Attribution License, which permits unrestricted use, distribution, and reproduction I NTER NATIONAL LIBRARY in any medium, provided the original author and source are credited.
} 
of heart failure in the United States will be nearly $\$ 70$ billion in the near future.

\section{Low Ventricular Pump Function}

Poor left ventricular contractility lead to impaired ejection of blood. Systolic dysfunction may be seen in children with structurally normal hearts such as ventricular non-compaction. In frequently, pharmacologic therapy is used in these particular patients. Drug choice and/or hospitalisations dependent on the severity of heart failure. Angiotensin-converting enzyme or its receptor blockers, diuretics, and beta-blockers are used. In children with stage IV cardiac failure resistant to pharmacologic treatment, invasive interventions such as intubation, mechanical circulatory support (ECMO) can be used for bridge to heart transplantation.

\section{Volume Overload with Normal Ventricular Pump Function}

Volume overload mab occur due to a intra- or extracardiac left-to-right shunt from the systemic to the pulmonary circulation (ventricular septal defect, atrioventricular canal defects, valvular failure). Surgical or catheter-based interventions to correct these defects lead to resolution of heart failure after stabilization of patients.

\section{Heart Failure Due to Pressure Overload with Normal Ventricular Pump Function}

Pressure overload may lead to heart failure. If there is a ventricular outflow obstruction which decrease left ventricular ejection. Resulting in inadequate cardiac output (e.g. aortic stenosis), or if ventricular hypertrophy due to prolonged pressure overload results in high filling pressures and congestion (e.g. hypertrophic cardiomyopathy). Surgical or catheter-based interventions to correct these defects lead to resolution of heart failure. Medical therapy may be needed for stabilization or symptom relief while awaiting a more definitive intervention.

New Pharmacologic Agents for the Treatment of Heat Failure

\section{Pulmonary vasodilators}

Sildenafil is a phosphodiesterase- 5 inhibitor. It is a pulmonary vasodilator, and use for treating improved ventricle. It is corrected quality of life in patients with impaired ventricular function. In frequently, it decreassed pulmonary hypertension and increased functional capacity $[1,2]$.

\section{For advanced heart failure}

Intravenous diuretics and inotropic(s) are frequently used in class IV heart failure.

\section{Inotropes and inodilators}

In patients with low out-put syndrome, inotropic(s) and/or inodilator, such as levosimendan, are used to treat heart failure prior to bridge to TX. Intracellular cyclic adenylate monophosphate levels, either by increased production (catecholamines) or by decreased degradation (phosphodiesterase III inhibition).

\section{Catecholamines}

Catecholamine agents, adrenaline or noradrenaline, improves myocardial contractility and may have an additional beneficial effect on peripheral vascular beds $[3,4]$.

Dopamine is the first preferred agent in children with severely impaired ventricle. These can be use using a combination with milrinone.

Dobutamine has the additive effect of reducing afterload and end-organ perfusion. Urine output, serum lactate, and mixed venous saturations increased milrinone.

Milrinone, as a phosphodiesterase III inhibitor, is the preferred drug in decompensated heart failure, as it increases contractility and reduces afterload without a significant increase in myocardial oxygen consumption $[4,5]$.

A randomized, double-blind, placebo-controlled trial in pediatric postoperative cardiac surgery patients demonstrated that children treated with high-dose milrinone infusion $(0.75 \mathrm{mcg} / \mathrm{kg} / \mathrm{min})$ were at a lower risk for the development of low cardiac output syndrome (LCOS) compared with children treated with placebo (12 versus 26 percent) [6].

To avoid hypotension, milrinone is initially administered as an infusion starting at a dose of $0.25 \mathrm{mcg} / \mathrm{kg} /$ min (without a pre-infusion bolus) and titrated upwards slowly as needed to a maximum dose of $1 \mathrm{mcg} / \mathrm{kg} / \mathrm{min}$.

Although milrinone is usually used in hospitalized children with heart failure refractory to oral drug therapy awaiting heart transplantation, a small study of 14 patients reported the safe administration of intravenous milrinone at home in children awaiting heart transplants [7]. Several centers (including ours) use home milrinone in selected children awaiting heart transplantation. In our practice, home milrinone infusion therapy is used in children who are clinically stable without end-organ dysfunction, with no history of arrhythmias, who generally are on a milrinone dose $\leq 0.5 \mathrm{mcg} / \mathrm{kg} / \mathrm{min}$ and a stable regimen of oral diuretic therapy, and who are under continuous adult supervision.

\section{Nesiritide}

Nesiritide is a recombinant B-type natriuretic peptide that reduces preload and afterload by promoting diuresis, natriuresis, and arterial- and veno-dilation, thereby improving cardiac output without a direct inotropic effect on the myocardium. It has been reported to be well tolerated and efficacious in children as illustrated in a prospective, open-label study in 63 children with refrac- 
tory heart failure that showed nesiritide was associated with improved urine output, serum creatinine, and cardiac function $[8,9]$. However, trials in adults with acute decompensated heart failure have failed to show that nesiritide is associated with lower mortality or rehospitalization rate, or that it improves dyspnea. In addition, there is an associated increased risk of hypotension. As a result, nesiritide is not recommended for general use in acute heart failure. (See "Nesiritide in the treatment of acute decompensated heart failure").

\section{Nonpharmacologic interventions for advanced heart failure}

Therapeutic interventions for selected patients with advanced heart failure refractory to pharmacologic therapy (stage D) may include:

\section{Positive pressure ventilation and ECMO (Extracor- poreal Membrane Oxygenation)}

Mechanical circulatory support such as ECMO is used in patients with end-stage heart failure in postcardiotomy low out-put syndrome. Unfortunately, morbidity and mortality is high, yet. The use of ECMO as a short-term device or centrifugal blood pumps increased the wait time by approximately one month. In most cases, the implant was performed later, when there was already impaired renal and/or liver function.

\section{Left Ventricular Assit Device and Heart transplan- tation}

Cardiac transplantion (HTX) is the most effective way for treatment of severe cardiac failure, although it is limited by the number of donors in pediatric patients. Pediatric post-HTX survival in the first year is approximately $90 \%$ and $60 \%$ over 10 years $[10,11]$. The major limitation for transplant is related to number of donors. Difficulty in providing organs varies according to variables such as blood type, age, the weight of recipient, and geographic distribution [12-15].

A large number of children with end-stage cardiomyopathy which required vasoactive drugs, visited the emergency departments of specialized hospitals. Therefore, to bridge to HTX left ventricular assist device is used in this severe clinical condition.

\section{References}

1. Guazzi M, Vicenzi M, Arena R, Guazzi MD (2011) PDE5 inhibition with sildenafil improves left ventricular diastolic function, cardiac geometry, and clinical status in patients with stable systolic heart failure: results of a 1-year, prospective, randomized, placebo-controlled study. Circ Heart Fail 4: 8-17.

2. Reinhardt Z, Uzun O, Bhole V, Ofoe V, Wilson D, et al. (2010) Sildenafil in the management of the failing Fontan circulation. Cardiol Young 20: 522-525.

3. Schweigmann U, Meierhofer C (2008) Strategies for the treatment of acute heart failure in children. Minerva Cardioangiol 56: 321-333.

4. Wessel DL (2001) Managing low cardiac output syndrome after congenital heart surgery. Crit Care Med 29: S220-S230.

5. Hoffman TM, Wernovsky G, Atz AM, Kulik TJ, Nelson DP, et al. (2003) Efficacy and safety of milrinone in preventing low cardiac output syndrome in infants and children after corrective surgery for congenital heart disease. Circulation 107: 996-1002.

6. Chandler HK, Kirsch R (2016) Management of the low cardiac syndrome following surgery for congenital heart disease. Curr Cardiol Rev 12: 107-111.

7. Price JF, Jeewa A, Denfield SW (2016) Clinical Characteristics and Treatment of Cardiomyopathies in Children. Curr Cardiol Rev 12: 85-98.

8. Gong B, Wu Z, Li Z (2016) Efficacy and safety of nesiritide in patients with decompensated heart failure: a meta-analysis of randomised trials. BMJ Open 6: e008545.

9. Mohammed SF, Korinek J, Chen HH, Burnett JC, Redfield MM (2008) Nesiritide in acute decompensated heart failure: current status and future perspectives. Rev Cardiovasc Med 9: 151-158.

10. Dipchand AI, Kirk R, Mahle WT, Tresler MA, Naftel DC, et al. (2013) Ten yr of pediatric heart transplantation: areport from the Pediatric Heart Transplant Study. Pediatr Transplant 17: 99-111.

11. Kirk R, Dipchand AI, Edwards LB, Kucheryavaya AY, Benden C, et al. (2012) The registry of the International Society for Heart and Lung Transplantation: fifteenth pediatric heart transplantation report-2012. J Heart Lung Transplant 31: 1065-1072.

12. Davies RR, Haldeman S, Pizarro C (2013) Regional variation in survival before and after pediatric heart transplantation-an analysis of the UNOS database. Am J Transplant 13: 1817-1829.

13. Mahle WT, Webber SA, Cherikh WS, Edwards LB (2012) Less urgent (UNOS 1B and 2) listings in pediatric heart transplantation: a vanishing breed. J Heart Lung Transplant 31: 782-784.

14. Almond CS, Thiagarajan RR, Piercey GE, Gauvreau K, Blume ED, et al. (2009) Waiting list mortality among children listed for heart transplantation in the United States. Circulation 119: 717-727.

15. Frey S, Pretre R, Stiasny B, Noll G, Ruschitzka F, et al. (2011) Morbidity and mortality in children and adolescents listed for heart transplantation. Cardiovasc Med 14: 176-181. 